\title{
STABILITY ANALYSIS OF RESOURCE-CONSUMER DYNAMIC MODELS
}

\author{
V. SREE HARI RAO ${ }^{\varpi 1}$ and P. RAJA SEKHARA RAO ${ }^{2}$
}

(Received 4 April, 2004; revised 16 December, 2005)

\begin{abstract}
A nutrient-consumer model involving a distributed delay in material recycling and a discrete delay in growth response has been analysed. Various easily verifiable sets of sufficient conditions for global asymptotic stability of the positive equilibrium solution of the model equations have been obtained and the length of the delay in each case has been estimated.
\end{abstract}

2000 Mathematics subject classification: $34 \mathrm{~K} 20,34 \mathrm{~K} 15,92 \mathrm{~A} 15$.

Keywords and phrases: nutrient-consumer dynamics.

\section{Introduction}

In the present paper, we analyse a mathematical model in which a biotic species such as micro algae or fungi feeds on a limiting nutrient (for example, nitrogen or phosphorous or vitamin $B_{12}$ ) in a cultured environment. That is, we wish to study the influence of the limited supply of a single nutrient on the growth of the consumer (biotic species) assuming that all other factors that influence the growth are managed, so as not to disturb the system. Many mathematical models have been proposed to explain the biological process involved in limited nutrient-consumer dynamics. One such popular model is a chemostat model. The name chemostat is associated with a laboratory device in which a continuously cultured environment is created to study the behaviour of the species with the supply of the growth-limiting nutrient under the control of the experimenter. The predictions made by the analysis of the chemostat models may be tested in this device, thus establishing a formidable link between mathematics (theory) and biology (experiment). In [31] it is also highlighted that a

\footnotetext{
${ }^{1}$ Department of Mathematics, Jawaharlal Nehru Technological University, Hyderabad 500072 , India; e-mail: vshrao@yahoo.com.

${ }^{2}$ Department of Mathematics, Government Polytechnic, Gudur 524 102, India; e-mail: raoprs@yahoo.com.

(C) Australian Mathematical Society 2006, Serial-fee code 1446-181 1/06
} 
chemostat is the case where "mathematics precedes the experiment". A fundamental mathematical model describing the growth in a chemostat is given by

$$
\begin{aligned}
& \frac{d x(t)}{d t}=D x_{0}-D x(t)-a U(x(t)) y(t), \\
& \frac{d y(t)}{d t}=a U(x(t)) y(t)-D y(t) .
\end{aligned}
$$

In (1.1), $x(t)$ denotes the growth-limiting nutrient and $y(t)$ denotes the biotic species feeding on $x$ at any time $t$.

Here $x_{0}$ is the input nutrient concentration which is a positive constant, measured in $\mathrm{mol} / \mathrm{vol}$. It denotes the quantity of nutrient available within the system at any time. The positive constant $D>0$ is the rate at which the nutrient is supplied and also the rate at which the contents of the growth medium are removed. It is popularly known as the washout rate and is measured in $1 / \mathrm{sec}$. Here $a>0$ is the maximal consumption rate of the nutrient and also the maximum specific growth rate of the microorganisms. This choice implies that the consumption means growth here. We note that $U$ is the functional response of the microorganisms describing how the nutrient is consumed by the species and is usually called the uptake function. For more details on chemostat models and the early development of them, we refer the readers to Smith and Waltman [31].

As a further development, the chemostat is seen as a replica of a simple lake. However there are some basic differences between the two. The main point that differentiates a lake from a chemostat is the inflow/outflow rate (washout rate). The washout rate in a chemostat is usually very high whereas in a lake it is low for most of the year. If one tries to treat the chemostat as a model of a natural lake, one should study the growth of microorganisms under a low washout rate. Due to this low washout (inflow), the nutrient becomes scarce which is a major survival problem for the species. One may even expect an extinction of the species. But observations (see $[5,9,29]$ and references therein) show that the species survive in spite of the low availability of nutrient. But the species growth is found to be low and oscillatory. To account for this oscillatory behaviour, mathematicians proposed different phenomena in the models such as periodic nutrient input or periodic inflow rate or a combination of both. But most mathematicians preferred the introduction of time delays in the growth response of the microorganisms to nutrient uptake in the models as a means of understanding the oscillatory behaviour. Freedman, So and Waltman [16] introduced time delays in a chemostat model with a discrete delay in the growth response of the consumer species and obtained conditions for the coexistence of competing species. We refer the readers to Ellermeyer [10] and Waltman [32] for some details on these models.

Again, when the washout rate is very small, the dead biomass in a lake resides there for a long time and hence there is every possibility of regeneration of nutrient due to 
bacterial decomposition of this dead biomass. It is natural to expect a time delay in the regeneration of nutrient due to bacterial decomposition. Nutrient-consumer models incorporating a distributed delay in nutrient recycling have been extensively studied in Beretta, Bischi and Solimano [2] and Freedman and Xu [18] (see also [5-7, 12, 19]).

These models describing the growth of microorganisms in a lake may be termed as chemostat-like models as they may be viewed as modifications of chemostat models (see [25] for a good account of these models).

Chemostat-like models with distributed time delays both in growth response and material recycling have received much attention in recent years (see $[1,5-7,19,20,22$ $30,33,34])$. The models described above feature either discrete delays or distributed delays but none has considered the combination of these two features. According to Nisbet and Gurney [21], the experimental data encourages the introduction of a discrete delay in the growth response of the consumer species. Further, when the delays are small, systems with constant fixed delays serve as good approximations to those with distributed delays. This prompts us to study the influence of the presence of a discrete delay in the growth response of the microorganisms in a chemostat model. Since the residence time of dead biomass in the system is measured on a timescale of years and because of the slow, continuous process of decomposition of the dead biomass, a distributed time lag seems more appropriate for nutrient recycling ([5]).

The model under consideration is based on the following biological principles:

- There is an external source from which the nutrient is supplied to the system. In the model, $x_{0}$ denotes the input nutrient concentration and $D$ denotes the rate at which it is supplied.

- There is an outlet to the system from which the nutrient and the consumer species are removed (washed out) at the same rate $D$.

- The consumer species consume the nutrient continuously at a constant rate $a$ and the consumption is nonlinearly related to the nutrient. Further, the consumption reaches a saturation level which means that unlimited supply of nutrient does not imply unlimited consumption. In our model the function $U$ represents this consumption.

- The growth of the consumer species is a delayed process while death is instantaneous.

- The growth of the consumer species is governed by the finite carrying capacity of the environment. The term $d$ represents the death rate due to this finite carrying capacity so that $1 / d$ denotes the carrying capacity.

- The consumer biotic species are subject to a natural death besides a washout. The constant $\gamma$ represents this death rate.

- The nutrient population is enriched by the recycling of a portion $b$ of the dead biomass of the consumer and this process is not instantaneous.

In the present paper, we estimate the length of the delay for which the positive 
equilibrium solution of the model equations is asymptotically stable, employing a Lyapunov functional technique. This, of course, provides a lower bound for the length of delay for which the stability of the system is guaranteed. It is interesting to note that no linearisation of the nonlinearities has taken place. Thus our study is not merely a local stability analysis of the model.

A chemostat-like model with a distributed time delay in material recycling and a discrete delay in growth response has been studied by Ruan ([29]) and He and Ruan ([19]). We wish to consider, for the first time, the influence of the finite carrying capacity of the environment in a model with a distributed time delay in nutrient recycling and a discrete time delay in the growth response of the consumer species. The model we study in this paper is fairly general in the sense that the models described in $[1-7,16,18]$ and $[19,29]$ become special cases of this model as we shall see in the coming sections. We obtain five sets of easily verifiable sufficient conditions for the global asymptotic stability of the positive equilibrium. It may be seen that our study includes fairly general classes of delay kernels and uptake functions.

The paper is organised as follows. Section 2 deals with the model. Further, basic properties of solutions of the model equations and the existence and uniqueness of equilibria have been discussed in this section. In Section 3, we obtain various easily verifiable sets of sufficient conditions for the global asymptotic stability of the positive equilibrium solution of the model equations. Global asymptotic stability of some important special cases is discussed in Section 4. Finally, a discussion follows in Section 5.

\section{The model and some preliminaries}

The above discussion yields the following system of equations describing the dynamics of the nutrient and the consumer populations:

$$
\begin{aligned}
& \frac{d x(t)}{d t}=D\left(x_{0}-x(t)\right)-a U(x(t)) y(t)+b \gamma \int_{0}^{\infty} f(s) y(t-s) d s, \\
& \frac{d y(t)}{d t}=-(\gamma+D) y(t)+c y(t) U(x(t-\tau))-d y^{2}(t),
\end{aligned}
$$

with the initial conditions

$$
x(s)=\phi_{1}(s), \quad y(s)=\phi_{2}(s), \quad-\infty<s \leq 0,
$$

where $\phi_{j}, j=1,2$, are nonnegative, bounded and continuous functions on $(-\infty, 0]$.

In (2.1), $x$ denotes the limiting nutrient and $y$ represents the microorganism feeding on $x$. Again $D>0$ is the constant washout rate of the biomass and the nutrient, while $x_{0}>0$ represents the constant nutrient input concentration. The positive constant $a$ 
represents the maximum uptake rate of the nutrient, while $0<c \leq a$ represents the maximum specific growth rate of the species $y$. Here $\gamma>0$ is the death-rate coefficient of $y$. The constant $b \in(0,1)$ represents the portion of the dead biomass recycled. The positive constant $d$ accounts for the finite carrying capacity of the environment. We note that $0 \leq \tau<\infty$ is the delay in the growth response of the consumer species. The function $U$ is known as the uptake function or response function of the species $y$ and represents how $y$ consumes $x$. A mathematical realisation of the third biological principle may lead us to the following assumptions on the uptake function $U$.

$\left(\mathrm{A}_{1}\right) \quad U(x)$ is a continuous, real-valued function defined on $R_{+}=[0, \infty)$ such that $U(0)=0, U(x)>0$ for $x>0$ and $\lim _{x \rightarrow \infty} U(x)=L_{1}<\infty$. It is clear that $U(x)$ is bounded and therefore there exists an $L>0$ such that $U(x) \leq L$ for all $x \in R_{+}$.

The delay kernel $f$ describes how the dead biomass is recycled and the mathematical imposition on $f$ is that it is a nonnegative function satisfying

$\left(\mathrm{A}_{2}\right) \int_{0}^{\infty} f(s) d s=1$ and $\int_{0}^{\infty} s f(s) d s<\infty$.

The second assumption on $f$ in $\left(\mathrm{A}_{2}\right)$ obviously implies that the average time delay in material recycling is finite.

Proceeding as in [6], it is easy to see that the system (2.1)-(2.2) has unique, continuable solutions on their maximal intervals of existence when $U$ satisfies a Lipschitz condition. However, conditions weaker than the Lipschitz condition on $U$, which ensure the existence and uniqueness of solutions have been presented in an earlier work of the authors [22]. Henceforth, it is tacitly assumed that the system $(2.1)-(2.2)$ has unique, continuable solutions. Further, proceeding as in $([6,22])$, it can be proved that the solutions of system (2.1) are nonnegative, bounded owing to the initial conditions (2.2) and that

$$
\begin{aligned}
& x(t) \leq \max \left\{x_{0}+\frac{b \gamma c L}{d D}, \sup _{-\infty<i \leq 0}\left\{\phi_{1}(t)\right\}\right\} \text { and } \\
& y(t) \leq \max \left\{\frac{c L}{d}, \sup _{-\infty<i \leq 0}\left\{\phi_{2}(t)\right\}\right\} .
\end{aligned}
$$

Now an equilibrium solution of $(2.1)$ is a solution of the system

$$
\begin{aligned}
& D x_{0}-D x-a U(x) y+b \gamma y=0, \\
& -(\gamma+D) y+c y U(x)-d y^{2}=0 .
\end{aligned}
$$

Clearly $\left(x_{0}, 0\right)$ is a solution of the system which is a partially feasible equilibrium of the system (2.1).

As in [22], it can be shown that a necessary and sufficient condition for the existence 
of a unique positive equilibrium solution $\left(x^{*}, y^{*}\right)$ for the system $(2.1)$ is that

$$
\frac{\gamma+D}{c}<U\left(x^{*}\right)<\frac{1}{2}\left\{\frac{\gamma+D}{c}+\frac{b \gamma}{a}+\sqrt{\left(\frac{\gamma+D}{c}-\frac{b \gamma}{a}\right)^{2}+\frac{4 d D}{a c} x_{0}}\right\} .
$$

Clearly $\left(x^{*}, y^{*}\right)$ satisfies

$$
D x^{*}+a U\left(x^{*}\right) y^{*}-b \gamma y^{*}=D x_{0} \text { and } c U\left(x^{*}\right)-d y^{*}=\gamma+D,
$$

or

$$
\begin{aligned}
x^{*} & =x_{0}-\frac{\left(a U\left(x^{*}\right)-b \gamma\right)\left(c U\left(x^{*}\right)-\gamma-D\right)}{d D}, \\
y^{*} & =\frac{c U\left(x^{*}\right)-\gamma-D}{d} .
\end{aligned}
$$

Hereafter, we tacitly assume that the system (2.1) has a unique positive equilibrium solution $\left(x^{*}, y^{*}\right)$ and confine ourselves to the study of this positive equilibrium solution. Also, we may establish that the existence of a unique positive equilibrium ensures the instability of $\left(x_{0}, 0\right)$ (see [22]).

It may be observed that system (2.1) is very general in the sense that many of the models studied earlier become special cases of it as we see below:

$\begin{array}{ll}\text { Choice of parameters } & \text { Models studied in } \\ \tau=0 & {[7]} \\ \tau=0, d=0 & {[2,6,7,18]} \\ \tau=0, d=0, f(s)=\delta(s) & {[1]} \\ \gamma=0, d=0, f(s)=\delta(s) & {[4,16]} \\ d=0 & {[19,29] .}\end{array}$

\section{Stability results}

In this section, we shall obtain various sets of sufficient conditions on the parameters that establish the asymptotic stability of the positive equilibrium solution $\left(x^{*}, y^{*}\right)$ of (2.1) and estimate the length of the delay for which these hold, employing a Lyapunov functional technique.

We recall that for the system

$$
X^{\prime}(t)=F(X),
$$

where $F$ is continuous in $X$ for $X \in \bar{G}$ and $G$ is an open set in $\mathbb{R}^{n}$, we have the following definition. 
Definition. $V(X)$ is a Lyapunov function in $G$ for (3.1) if $V^{\prime}=\nabla V \cdot F \leq 0$ on $G$.

Consider the system (for example, (2.1))

$$
X^{\prime}(t)=F\left(X_{t}\right)
$$

where $F$ is continuous in $X_{t}$ and defined by $X_{t}(s)=X(t+s), s \in(-\infty, 0]$ such that $F(0)=0$ for $X \in \bar{G}$ and $G$ is an open set in $\mathbb{R}^{n}$. Let $X_{H}(t)$ denote the set $B C(-\infty, 0]$ such that $\left\|X_{t}\right\| \leq H$. Now consider the autonomous system (*) with the initial conditions $\phi$ which are nonnegative, bounded and continuous functions on $(-\infty, 0]$ such that $\|\phi\| \leq H$. Then the following result holds.

THEOREM A ([8]). Let $V\left(X_{t}\right)$ be a scalar functional such that $V: X_{H}(t) \rightarrow[0, \infty)$ and it is uniform Lipschitz on $X_{H}(t)$ for some $H>0$. If, furthermore,

$$
w_{1}(\|X(t)\|) \leq V\left(X_{t}\right), \quad V(0)=0, \quad V_{(*)}^{\prime}\left(X_{t}\right) \leq-w_{2}(\|X(t)\|),
$$

for all $t \geq 0$, then the trivial equilibrium of (*) is globally (uniformly) asymptotically stable.

Here $w_{i}(s), i=1,2, s \geq 0$ are scalar continuous nondecreasing functions such that $w_{i}(0)=0, w_{i}(s)>0$ for $s>0$ and $\lim _{s \rightarrow \infty} w_{i}(s)=+\infty$.

The difficulty lies in finding a suitable Lyapunov functional that satisfies the conditions of Theorem A. There are no general rules for finding a Lyapunov functional, but usually it is convenient to construct it step by step as we see below.

Before proceeding further, we make the following change of variables:

$$
x_{1}(t)=x(t)-x^{*}, \quad y_{1}(t)=\frac{y(t)-y^{*}}{y^{*}}, \quad U_{1}\left(x_{1}(t)\right)=U(x(t))-U\left(x^{*}\right) .
$$

Then (2.1) after a rearrangement assumes the form

$$
\begin{aligned}
x_{1}^{\prime}(t)= & -D x_{1}(t)-a y^{*} U_{1}\left(x_{1}(t)\right)-a y^{*} U(x(t)) y_{1}(t) \\
& +b \gamma y^{*} \int_{0}^{\infty} f(s) y_{1}(t-s) d s, \\
y_{1}^{\prime}(t)= & \left(1+y_{1}(t)\right)\left[c U_{1}\left(x_{1}(t-\tau)\right)-d y^{*} y_{1}(t)\right],
\end{aligned}
$$

where ' denotes $d / d t$.

The following assumptions on the transformed uptake function $U_{1}$ are used in our subsequent discussion.

( $\left.\mathrm{A}_{3}\right) \quad U \in C^{1}[0, \infty)$ and there exists a positive constant $M_{1}$ such that $d U / d x<M_{1}$. In other words, $d U_{1} / d x_{1}<M_{1}$ for $x_{1} \in\left[-x^{*}, \infty\right)$. 
(A $\left.\mathrm{A}_{4}\right) x_{1} U_{1}\left(x_{1}\right)>0$ for all $x_{1} \neq 0$ and there exist positive constants $\alpha_{1}, \alpha_{2}$ and $\beta$ such that $\alpha_{1} \leq U_{1}\left(x_{1}\right) / x_{1} \leq \alpha_{2}$ for $x_{1} \geq-x^{*}$ and $\beta=\min _{x_{1} \geq-x^{*}}\left\{x_{1} / U_{1}\left(x_{1}\right)\right\}$.

The foregoing discussion explains the construction of a Lyapunov functional that will be useful in establishing our main results.

Consider the functional

$$
V_{1}(t) \equiv V_{1}\left(x_{1}(t), y_{1}(t)\right)=\frac{A}{2} x_{1}^{2}(t)+B\left[y_{1}(t)-\log \left(1+y_{1}(t)\right)\right],
$$

where $A$ and $B$ are positive constants which will be chosen appropriately.

The time derivative of $V_{1}$ along the solutions of (3.2) is given by

$$
\begin{aligned}
\frac{d V_{1}}{d t}= & A x_{1}(t)\left\{-D x_{1}(t)-a y^{*} U_{1}\left(x_{1}(t)\right)-a y^{*} U(x(t)) y_{1}(t)\right. \\
& \left.+b \gamma y^{*} \int_{0}^{\infty} f(s) y_{1}(t-s) d s\right\}+B y_{1}(t)\left\{c U_{1}\left(x_{1}(t-\tau)\right)-d y^{*} y_{1}(t)\right\} \\
\leq & -A\left(D-\frac{b \gamma y^{*}}{2}\right) x_{1}^{2}(t)-A a y^{*} U_{1}\left(x_{1}(t)\right) x_{1}(t) \\
& -A a y^{*} U(x(t)) x_{1}(t) y_{1}(t)+\frac{A b \gamma y^{*}}{2} \int_{0}^{\infty} f(s) y_{1}^{2}(t-s) d s \\
& +B c y_{1}(t) U_{1}\left(x_{1}(t-\tau)\right)-B d y^{*} y_{1}^{2}(t) .
\end{aligned}
$$

Now consider

$$
\begin{aligned}
y_{1}(t) U_{1}\left(x_{1}(t-\tau)\right)= & y_{1}(t)\left[U_{1}\left(x_{1}(t)\right)-\int_{t-\tau}^{t} \frac{d}{d s}\left(U_{1}\left(x_{1}(s)\right) d s\right]\right. \\
= & y_{1}(t)\left[U_{1}\left(x_{1}(t)\right)-\int_{t-\tau}^{t}\left(\frac{d U_{1}\left(x_{1}(s)\right)}{d x_{1}(s)} \cdot \frac{d x_{1}(s)}{d s}\right) d s\right] \\
= & y_{1}(t) U_{1}\left(x_{1}(t)\right)-y_{1}(t) \int_{t-\tau}^{t}\left(\frac{d U_{1}\left(x_{1}(s)\right)}{d x_{1}(s)}\right) \\
& \times\left\{-D x_{1}(s)-a y^{*} U_{1}\left(x_{1}(s)\right)-a y^{*} U(x(s)) y_{1}(s)\right. \\
& \left.+b \gamma y^{*} \int_{0}^{\infty} f(s) y_{1}(s-u) d u\right\} d s \\
\leq & y_{1}(t) U_{1}\left(x_{1}(t)\right) \\
& +\frac{1}{2}\left[D+2 a y^{*}+b \gamma y^{*}\right] y_{1}^{2}(t) \int_{t-\tau}^{t}\left(\frac{d U_{1}\left(x_{1}(s)\right)}{d x_{1}(s)}\right)^{2} d s \\
& +\frac{D}{2} \int_{1-\tau}^{t} x_{1}^{2}(s) d s+\frac{a y^{*}}{2} \int_{t-\tau}^{t} U_{1}^{2}\left(x_{1}(s)\right) d s \\
& +\frac{a y^{*}}{2} \int_{t-\tau}^{t} U^{2}(x(s)) y_{1}^{2}(s) d s
\end{aligned}
$$




$$
+\frac{b \gamma y^{*}}{2} \int_{t-\tau}^{t} \int_{0}^{\infty} f(u) y_{1}^{2}(s-u) d u d s
$$

Using this in (3.4), we get

$$
\begin{aligned}
\frac{d V_{1}}{d t} \leq & -A\left(D-\frac{b \gamma y^{*}}{2}\right) x_{1}^{2}(t)-A a y^{*} U(x(t)) x_{1}(t) y_{1}(t) \\
& +B c U_{1}\left(x_{1}(t)\right) y_{1}(t)-B\left[d y^{*}-\frac{c}{2}\left(D+2 a y^{*}+b \gamma y^{*}\right) M \tau\right] y_{1}^{2}(t) \\
& -A a y^{*} U_{1}\left(x_{1}(t)\right) x_{1}(t)+A \frac{b \gamma y^{*}}{2} \int_{0}^{\infty} f(s) y_{1}^{2}(t-s) d s \\
& +\frac{B c D}{2} \int_{t-\tau}^{t} x_{1}^{2}(s) d s+B \frac{a y^{*} c}{2} \int_{t-\tau}^{t} U_{1}^{2}\left(x_{1}(s)\right) d s \\
& +B \frac{a y^{*} c}{2} \int_{t-\tau}^{t} U^{2}(x(s)) y_{1}^{2}(s) d s \\
& +B \frac{b \gamma y^{*} c}{2} \int_{t-\tau}^{t} \int_{0}^{\infty} f(u) y_{1}^{2}(s-u) d u d s
\end{aligned}
$$

using $\left(\mathrm{A}_{3}\right)$ and denoting $M=M_{1}{ }^{2}$. Now consider the functional

$$
\begin{aligned}
V_{2}(t) \equiv & V_{2}\left(x_{1}(t), y_{1}(t)\right) \\
= & \frac{B c}{2} \int_{t-\tau}^{t} \int_{s}^{t}\left\{D x_{1}^{2}(\nu)+a y^{*} U_{1}^{2}\left(x_{1}(\nu)\right)+a y^{*} U^{2}(x(\nu)) y_{1}^{2}(\nu)\right. \\
& \left.+b \gamma y^{*} \int_{0}^{\infty} f(u) y_{1}^{2}(v-u) d u\right\} d \nu .
\end{aligned}
$$

Then the time derivative of $V_{2}$ is given by

$$
\begin{aligned}
\frac{d V_{2}(t)}{d t}= & \frac{B c}{2}\left\{D x_{1}^{2}(t)+a y^{*} U_{1}^{2}\left(x_{1}(t)\right)+a y^{*} U^{2}(x(t)) y_{1}^{2}(t)\right. \\
& \left.+b \gamma y^{*} \int_{0}^{\infty} f(u) y_{1}^{2}(t-u) d u\right\} \tau \\
& -\frac{B c}{2} \int_{t-\tau}^{t}\left\{D x_{1}^{2}(s)+a y^{*} U_{1}^{2}\left(x_{1}(s)\right)+a y^{*} U^{2}(x(s)) y_{1}^{2}(s)\right. \\
& \left.+b \gamma y^{*} \int_{0}^{\infty} f(u) y_{1}^{2}(s-u) d u\right\} d s
\end{aligned}
$$

Now from (3.5) and (3.6), we have

$$
\frac{d V_{1}}{d t}+\frac{d V_{2}}{d t} \leq-A\left(D-\frac{b \gamma y^{*}}{2}\right) x_{1}^{2}(t)-A a y^{*} U(x(t)) x_{1}(t) y_{1}(t)
$$




$$
\begin{aligned}
& -B\left[d y^{*}-\frac{c}{2}\left(D+2 a y^{*}+b \gamma y^{*}\right) M \tau\right] y_{1}^{2}(t) \\
& -A a y^{*} U_{1}\left(x_{1}(t)\right) x_{1}(t)+A \frac{b \gamma y^{*}}{2} \int_{0}^{\infty} f(s) y_{1}^{2}(t-s) d s \\
& +B c U_{1}\left(x_{1}(t)\right) y_{1}(t) \\
& +\frac{B c}{2} \tau\left\{D x_{1}^{2}(t)+a y^{*} U_{1}^{2}\left(x_{1}(t)\right)+a y^{*} U^{2}(x(t)) y_{1}^{2}(t)\right\} \\
& +\frac{B c}{2} b \gamma y^{*} \tau \int_{0}^{\infty} f(u) y_{1}^{2}(t-u) d u .
\end{aligned}
$$

Now define the functional

$$
V_{3}(t) \equiv V_{3}\left(x_{1}(t), y_{1}(t)\right)=[A+B c \tau] \frac{b \gamma y^{*}}{2} \int_{0}^{\infty} f(s) \int_{t-s}^{t} y_{1}^{2}(z) d z d s
$$

The time derivative of $V_{3}$ is given by

$$
\frac{d V_{3}}{d t}=[A+B c \tau] \frac{b \gamma y^{*}}{2}\left[y_{1}^{2}(t)-\int_{0}^{\infty} f(s) y_{1}^{2}(t-s) d s\right] .
$$

We now define our main Lyapunov functional

$$
V(t) \equiv V\left(x_{1}(t), y_{1}(t)\right)=V_{1}(t)+V_{2}(t)+V_{3}(t)
$$

Then clearly $V(0,0)=0$ and $V\left(x_{1}(t), y_{1}(t)\right)>0$ for $x_{1}(t)>0$ and $y_{1}(t)>0$. Using (3.7) and (3.8), the time derivative of $V$ along the solutions of (3.2) is given by

$$
\begin{aligned}
\frac{d V}{d t} \leq & -A\left(D-\frac{b \gamma y^{*}}{2}\right) x_{1}^{2}(t)-A a y^{*} U(x(t)) x_{1}(t) y_{1}(t) \\
& -B\left[d y^{*}-\frac{c}{2}\left(D+2 a y^{*}+b \gamma y^{*}\right) M \tau\right] y_{1}^{2}(t) \\
& -A a y^{*} U_{1}\left(x_{1}(t)\right) x_{1}(t)+B c U_{1}\left(x_{1}(t)\right) y_{1}(t) \\
& +\frac{B c}{2}\left[D x_{1}^{2}(t)+a y^{*} U_{1}^{2}\left(x_{1}(t)\right)+a y^{*} U^{2}(x(t)) y_{1}^{2}(t)\right] \tau+[A+B c \tau] \frac{b \gamma y^{*}}{2} y_{1}^{2}(t) \\
= & -\left[A\left(D-\frac{b \gamma y^{*}}{2}\right)-\frac{B c}{2} D \tau\right] x_{1}^{2}(t)-\left\{B d y^{*}-A \frac{b \gamma y^{*}}{2}\right. \\
& \left.-\frac{B c}{2}\left[\left(D+2 a y^{*}+b \gamma y^{*}\right) M+b \gamma y^{*}+a y^{*} U^{2}(x(t))\right] \tau\right\} y_{1}^{2}(t) \\
& -A a y^{*} U(x(t)) x_{1}(t) y_{1}(t)+B c\left(\frac{U_{1}\left(x_{1}(t)\right)}{x_{1}(t)}\right) x_{1}(t) y_{1}(t) \\
& -A a y^{*} U_{1}\left(x_{1}(t)\right) x_{1}(t)+\frac{B c a y^{*}}{2} \tau U_{1}^{2}\left(x_{1}(t)\right) .
\end{aligned}
$$


Case I Let $\left(D-b \gamma y^{*} / 2\right)>0$ and $B d y^{*}-A b \gamma y^{*} / 2>0$.

Now inequality (3.9) upon further simplification and rearrangement yields

$$
\begin{aligned}
\frac{d V}{d t} \leq & -\left\{\left[A\left(D-\frac{b \gamma y^{*}}{2}\right)-\frac{B c D}{2} \tau\right] x_{1}^{2}(t)+\left[A a y^{*} U(x(t))-B c \alpha_{2}\right] x_{1}(t) y_{1}(t)\right. \\
& +\left[B d y^{*}-A \frac{b \gamma y^{*}}{2}\right. \\
& \left.\left.-\frac{B c}{2} \tau\left[\left(D+2 a y^{*}+b \gamma y^{*}\right) M+b \gamma y^{*}+a y^{*} U^{2}(x(t))\right]\right] y_{1}^{2}(t)\right\} \\
& -\left[A a y^{*} \beta-\frac{B c}{2} a y^{*} \tau\right] U_{1}^{2}\left(x_{1}(t)\right),
\end{aligned}
$$

where $\alpha_{2}$ and $\beta$ are as defined in $\left(\mathrm{A}_{4}\right)$.

Then $d V / d t$ is negative definite if

$$
A a y^{*} \beta>\frac{B C}{2} a y^{*} \tau \text { and } C_{1}^{2}<\left(A_{1}-B_{1} \tau\right)\left(A_{2}-B_{2} \tau\right),
$$

where $2 C_{1}=A a y^{*} L-B c \alpha_{2}, A_{1}=A\left(D-b \gamma y^{*} / 2\right), B_{1}=B c D / 2, A_{2}=B d y^{*}-$ $A b \gamma y^{*} / 2$ and $\left.B_{2}=B c\left[\left(D+2 a y^{*}+b \gamma y^{*}\right) M+b \gamma y^{*}+a y^{*} L^{2}\right)\right] / 2$, using that $U(x) \leq L$.

Now the second inequality of (3.10) implies that

$$
B_{1} B_{2} \tau^{2}-\left(A_{1} B_{2}+A_{2} B_{1}\right) \tau+A_{1} A_{2}-C_{1}^{2}>0 .
$$

Consider

$$
B_{1} B_{2} \tau^{2}-\left(A_{1} B_{2}+A_{2} B_{1}\right) \tau+\left(A_{1} A_{2}-C_{1}^{2}\right)=0
$$

A necessary condition for the second inequality in (3.10) to hold is that $C_{1}^{2}<A_{1} A_{2}$. This condition implies that both the roots of (3.11) are real, distinct and positive, since $A_{1}, A_{2}, B_{1}$ and $B_{2}$ are all positive.

Let $\tau_{1}$ and $\tau_{2}$ represent the two roots of (3.11) and without loss of generality, let $\tau_{1}<\tau_{2}$. Clearly, $\tau_{1} \leq A_{1} / B_{1} \leq \tau_{2}$ and $\tau_{1} \leq A_{2} / B_{2} \leq \tau_{2}$. Therefore, if we choose $\tau^{*}=\min \left\{\tau_{1}, 2 A \beta / B C\right\}$ then $d V / d t$ is negative definite for $0 \leq \tau \leq \tau^{*}$ where

$$
\tau_{1}=\frac{1}{2}\left(\frac{A_{1}}{B_{1}}+\frac{A_{2}}{B_{2}}-\sqrt{\left(\frac{A_{1}}{B_{1}}-\frac{A_{2}}{B_{2}}\right)^{2}+\frac{4 C_{1}^{2}}{B_{1} B_{2}}}\right) .
$$

We now consider the following case. 
Case II Let $A\left(D-b \gamma y^{*} / 2-\left(a y^{*} / 2\right) L\right)-B c \alpha_{2} / 2>0$ and $B d y^{*}-A b \gamma y^{*} / 2-$ $\left(\right.$ Aay $\left.^{*} / 2\right) L-B c \alpha_{2} / 2>0$.

We now observe that inequality (3.9) may be simplified as

$$
\begin{aligned}
\frac{d V}{d t} \leq & -\left\{A\left(D-\frac{b \gamma y^{*}}{2}\right)-\frac{A a y^{*}}{2} U(x(t))-\frac{B c \alpha_{2}}{2}-\frac{B c D}{2} \tau\right\} x_{1}^{2}(t) \\
& -\left\{B d y^{*}-\frac{A b \gamma y^{*}}{2}-\frac{A a y^{*}}{2} U(x(t))-\frac{B c \alpha_{2}}{2}\right. \\
& \left.-\frac{B c \tau}{2}\left[\left(D+2 a y^{*}+b \gamma y^{*}\right) M+b \gamma y^{*}+a y^{*} U^{2}(x(t))\right]\right\} y_{1}^{2}(t) \\
& -\left\{A a y^{*} \beta-\frac{B c}{2} a y^{*} \tau\right\} U_{1}^{2}\left(x_{1}(t)\right) .
\end{aligned}
$$

Then $d V / d t$ is negative definite if we choose $0 \leq \tau<\tau^{*}$, where

$$
\begin{aligned}
\tau^{*}=\min \{ & \frac{A\left(2 D-b \gamma y^{*}-a y^{*} L\right)-B c \alpha_{2}}{B c D}, \frac{2 A \beta}{B c}, \\
& \left.\frac{2 B d y^{*}-A b \gamma y^{*}-A a y^{*} L-B c \alpha_{2}}{B c\left[\left(D+2 a y^{*}+b \gamma y^{*}\right) M+b \gamma y^{*}+a y^{*} L^{2}\right]}\right\} .
\end{aligned}
$$

Upon eliminating the arbitrary constants $A$ and $B$ from the above, we obtain

$$
d y^{*}\left(2 D-b \gamma y^{*}-a y^{*} L\right)-D c \alpha_{2}>0,
$$

which gives a sufficient condition for the existence of $\tau^{*}>0$.

We now simplify (3.9) as

$$
\begin{aligned}
\frac{d V}{d t} \leq & -\left[A\left(D-\frac{b \gamma y^{*}}{2}\right)-\frac{B c D}{2} \tau\right] x_{1}^{2}(t) \\
& -\left[B d y^{*}-\frac{A b \gamma}{2} y^{*}\right. \\
& \left.-\frac{B c}{2}\left[M\left(D+2 a y^{*}+b \gamma y^{*}\right)+b \gamma y^{*}+a y^{*} L^{2}\right] \tau\right] y_{1}^{2}(t) \\
& -A a y^{*} U(x(t)) x_{1}(t) y_{1}(t)+B c \alpha_{2} x_{1}(t) y_{1}(t) \\
& -\left[A a y^{*}\left(\frac{U_{1}\left(x_{1}(t)\right)}{x_{1}(t)}\right)-\tau \frac{B c}{2} a y^{*}\left(\frac{U_{1}\left(x_{1}(t)\right)}{x_{1}(t)}\right)^{2}\right] x_{1}^{2}(t) \\
\leq & -\left[A\left(D-\frac{b \gamma y^{*}}{2}+a y^{*} \alpha_{1}\right)-\frac{B c}{2}\left(D+a y^{*} \alpha_{2}^{2}\right) \tau\right] x_{1}^{2}(t) \\
& -\left[A a y^{*} U(x(t))-B c \alpha_{2}\right] x_{1}(t) y_{1}(t) \\
& -\left[B d y^{*}-\frac{A b \gamma y^{*}}{2}\right.
\end{aligned}
$$




$$
\left.-\frac{B c}{2}\left[M\left(D+2 a y^{*}+b \gamma y^{*}\right)+b \gamma y^{*}+a y^{*} L^{2}\right] \tau\right] y_{1}^{2}(t) \text {. }
$$

We now consider a third case.

Case III Let $A\left(D-b \gamma y^{*} / 2+a y^{*} \alpha_{1}-a y^{*} L / 2\right)-B c \alpha_{2} / 2>0$ and $B d y^{*}-$ $A b \gamma y^{*} / 2-A a y^{*} L / 2-B c \alpha_{2} / 2>0$.

Now upon using the inequality $x y \leq\left(x^{2}+y^{2}\right) / 2$ and rearranging, (3.12) assumes the form

$$
\begin{aligned}
\frac{d V}{d t} \leq & -\left[A\left(D-\frac{b \gamma y^{*}}{2}+a y^{*} \alpha_{1}-\frac{a y^{*}}{2} U(x)\right)-\frac{B c \alpha_{2}}{2}-\frac{B c}{2}\left(D+a y^{*} \alpha_{2}^{2}\right) \tau\right] x_{1}^{2}(t) \\
& -\left[B d y^{*}-A \frac{b \gamma}{2} y^{*}-\frac{A}{2} a y^{*} U(x)-\frac{B c}{2} \alpha_{2}\right. \\
& \left.-\frac{B c}{2} \tau\left[M\left(D+2 a y^{*}+b \gamma y^{*}\right)+b \gamma y^{*}+a y^{*} L^{2}\right]\right] y_{1}^{2}(t) .
\end{aligned}
$$

Therefore, for $0 \leq \tau<\tau^{*}=\min \left\{m_{1}, m_{2}\right\}$, where

$$
\begin{aligned}
& m_{1}=\frac{A\left(2 D-b \gamma y^{*}+2 a y^{*} \alpha_{1}-a y^{*} L\right)-B c \alpha_{2}}{B c\left(D+a y^{*} \alpha_{2}^{2}\right)}, \\
& m_{2}=\frac{2 B d y^{*}-A b \gamma y^{*}-A a y^{*} L-B c \alpha_{2}}{B c\left[M\left(D+2 a y^{*}+b \gamma y^{*}\right)+b \gamma y^{*}+a y^{*} L^{2}\right]},
\end{aligned}
$$

we have $d V / d t<0$.

Case IV Let $D-b \gamma y^{*} / 2+a y^{*} \alpha_{1}>0$ and $B d y^{*}-A b \gamma y^{*} / 2>0$.

Now from (3.12), we have $d V / d t<0$ provided

$$
C_{1}^{\prime 2}<\left(A_{1}^{\prime}-B_{1}^{\prime} \tau\right)\left(A_{2}^{\prime}-B_{2}^{\prime} \tau\right)
$$

that is, $B_{1}^{\prime} B_{2}^{\prime} \tau^{2}-\left(A_{1}^{\prime} B_{2}^{\prime}+A_{2}^{\prime} B_{1}^{\prime}\right) \tau+\left(A_{1}^{\prime} A_{2}^{\prime}-C_{1}^{\prime 2}\right)>0$, in which

$$
\begin{aligned}
& A_{1}^{\prime}=A\left(D-b \gamma y^{*} / 2+a y^{*} \alpha_{1}\right), \quad B_{1}^{\prime}=\frac{B c}{2}\left(D+a y^{*} \alpha_{2}^{2}\right), \\
& C_{1}^{\prime}=\frac{1}{2}\left(A a y^{*} L-B c \alpha_{2}\right), \quad A_{2}^{\prime}=B d y^{*}-\frac{A b \gamma y^{*}}{2} \text { and } \\
& B_{2}^{\prime}=\frac{B c}{2}\left[M\left(D+2 a y^{*}+b \gamma y^{*}\right)+b \gamma y^{*}+a y^{*} L^{2}\right] .
\end{aligned}
$$

Arguing as in Case I, it can be shown that the equation

$$
B_{1}^{\prime} B_{2}^{\prime} \tau^{2}-\left(A_{1}^{\prime} B_{2}^{\prime}+A_{2}^{\prime} B_{1}^{\prime}\right) \tau+\left(A_{1}^{\prime} A_{2}^{\prime}-C_{1}^{\prime 2}\right)=0
$$


has two distinct, positive, real roots, say, $\tilde{\tau}_{1}, \tilde{\tau}_{2}\left(>\tilde{\tau}_{1}\right)$. Further, it is easy to see that $\tilde{\tau}_{1} \leq A_{1}^{\prime} / B_{1}^{\prime} \leq \tilde{\tau}_{2}$ and $\tilde{\tau}_{1} \leq A_{2}^{\prime} / B_{2}^{\prime} \leq \tilde{\tau}_{2}$. Therefore, for

$$
\tau^{*}=\tilde{\tau}_{1}=\frac{1}{2}\left(\frac{A_{1}^{\prime}}{B_{1}^{\prime}}+\frac{A_{2}^{\prime}}{B_{2}^{\prime}}-\sqrt{\left(\frac{A_{1}^{\prime}}{B_{1}^{\prime}}-\frac{A_{2}^{\prime}}{B_{2}^{\prime}}\right)^{2}+\frac{4 C_{1}^{\prime 2}}{B_{1}^{\prime} B_{2}^{\prime}}}\right)
$$

we have $d V / d t<0$.

We are now in a position to state and prove our result which provides four different estimates of a lower bound for the length of time delay for which the equilibrium solution $(0,0)$ of $(3.2)$ and hence the positive equilibrium $\left(x^{*}, y^{*}\right)$ of $(2.1)$ is globally asymptotically stable.

THEOREM 3.1. Assume that the delay kernel $f$ satisfies $\left(\mathrm{A}_{2}\right)$ and the uptake function $U_{1}$ satisfies $\left(\mathrm{A}_{1}\right),\left(\mathrm{A}_{3}\right)$ and $\left(\mathrm{A}_{4}\right)$. The equilibrium solution $(0.0)$ of $(3.2)$ is globally asymptotically stable for $0 \leq \tau<\tau^{*}$, where $\tau^{*}$ may be estimated as follows with suitably chosen positive constants $A$ and $B$.

Case I. $\left(D-b \gamma y^{*} / 2\right)>0, B d y^{*}-A b \gamma y^{*} / 2>0$ and (3.10) hold. Then

$$
\tau^{*}=\min \left\{\frac{2 A \beta}{B c}, \frac{1}{2}\left(\frac{A_{1}}{B_{1}}+\frac{A_{2}}{B_{2}}-\sqrt{\left(\frac{A_{1}}{B_{1}}-\frac{A_{2}}{B_{2}}\right)^{2}+\frac{4 C_{1}^{2}}{B_{1} B_{2}}}\right)\right\},
$$

where

$$
A_{1}=A\left(D-\frac{b \gamma y^{*}}{2}\right), \quad B_{1}=\frac{B c D}{2}, \quad 2 C_{1}=A a y^{*} L-B c \alpha_{2}
$$

$$
A_{2}=B d y^{*}-\frac{A b \gamma y^{*}}{2} \quad \text { and } \quad B_{2}=\frac{B c}{2}\left[\left(D+2 a y^{*}+b \gamma y^{*}\right) M+b \gamma y^{*}+a y^{*} L^{2}\right] \text {. }
$$

Case II. Suppose $A\left(D-b \gamma y^{*} / 2-a y^{*} L / 2\right)-B c \alpha_{2} / 2>0$ and $B d y^{*}-A b \gamma y^{*} / 2-$ $\operatorname{Aay}^{*} L / 2-B c \alpha_{2} / 2>0$. Then

$$
\begin{aligned}
& \tau^{*}=\min \left\{\frac{A\left(2 D-b \gamma y^{*}-a y^{*} L\right)-B c \alpha_{2}}{B c D}, \frac{2 A \beta}{B c},\right. \\
& \left.\frac{2 B d y^{*}-A b \gamma y^{*}-A a y^{*} L-B c \alpha_{2}}{B c\left[\left(D+2 a y^{*}+b \gamma y^{*}\right) M+b \gamma y^{*}+a y^{*} L^{2}\right]}\right\} \text {. }
\end{aligned}
$$

Case III. Suppose $A\left(D+a y^{*} \alpha_{1} / 2-b \gamma y^{*} / 2-a y^{*} L / 2\right)-B c \alpha_{2} / 2>0$ and $B d y^{*}-$ $A b \gamma y^{*} / 2-A a y^{*} L / 2-B c \alpha_{2} / 2>0$. Then $\tau^{*}=\min \left\{m_{1}, m_{2}\right\}$, where

$$
\begin{aligned}
& m_{1}=\frac{A\left(2 D-b \gamma y^{*}+2 a y^{*} \alpha_{1}-a y^{*} L\right)-B c \alpha_{2}}{B c\left(D+a y^{*} \alpha_{2}^{2}\right)}, \\
& m_{2}=\frac{2 B d y^{*}-A b \gamma y^{*}-A a y^{*} L-B c \alpha_{2}}{B c\left[M\left(D+2 a y^{*}+b \gamma y^{*}\right)+b \gamma y^{*}+a y^{*} L^{2}\right]} .
\end{aligned}
$$


Case IV. Suppose $A\left(D+a y^{*} \alpha_{1} / 2-b \gamma y^{*} / 2\right)>0$ and $B d y^{*}-A b \gamma y^{*} / 2>0$. Then

$$
\tau^{*}=\frac{1}{2}\left(\frac{A_{1}^{\prime}}{B_{1}^{\prime}}+\frac{A_{2}^{\prime}}{B_{2}^{\prime}}-\sqrt{\left(\frac{A_{1}^{\prime}}{B_{1}^{\prime}}-\frac{A_{2}^{\prime}}{B_{2}^{\prime}}\right)^{2}+\frac{4 C_{1}^{\prime 2}}{B_{1}^{\prime} B_{2}^{\prime}}}\right),
$$

where $A_{1}^{\prime}=A\left(D-b \gamma y^{*} / 2+a y^{*} \alpha_{1}\right), B_{1}^{\prime}=B c\left(D+a y^{*} \alpha_{2}^{2}\right) / 2, C_{1}^{\prime}=C_{1}, A_{2}^{\prime}=A_{2}$ and $B_{2}^{\prime}=B_{2}$.

Proof. Since the negative definiteness of $d V / d t$ follows from each of the Cases IIV depending on which case $\tau^{*}$ is estimated from, it is easy to see that $V$, constructed above, is the required Lyapunov functional, and hence the conclusion of the theorem follows from standard arguments (see [22]).

The following example illustrates the above result.

EXAMPLE 3.2. Consider the system (2.1) with $D=1, a=4, b=0.2, \gamma=1$, $c=3$ and $d=20$. That is,

$$
\begin{aligned}
& x^{\prime}(t)=\left(x_{0}-x(t)\right)-4 U(x(t)) y(t)+(0.2) \int_{0}^{\infty} f(s) y(t-s) d s, \\
& y^{\prime}(t)=-2 y(t)+3 U(x(t-\tau))-20 y^{2}(t),
\end{aligned}
$$

in which $x_{0} \approx 9.68$ and $U(x)=x /(1+x)$. Clearly, $\left(x^{*}, y^{*}\right)=(8,1 / 30)$ and $U\left(x^{*}\right)=8 / 9$. Further, $M=1, \beta=9, \alpha_{2}=1 / 9$ and $\alpha_{1}=0.0122$, assuming the bound $x(t) \leq x_{0}+b \gamma c L / d D$, given in Section 2 .

Then for the choice of $A=1$ and $B=1$ we have, from Theorem 3.1,

(I) $\tau_{1}=0.3043, \tau_{2}=0.6724$ and $2 A \beta / B c=6$ and thus $\tau^{*}=0.3043$,

(II) $\tau^{*}=0.202$,

(III) $m_{1}=0.5093, m_{2}=0.203$ and thus $\tau^{*}=0.203$, and

(IV) $\tilde{\tau}_{1}=0.3055, \tilde{\tau}_{2}=0.673$ and $\tau^{*}=\tilde{\tau_{1}}=0.3055$.

We observe that the estimates on $\tau^{*}$ in these cases may be improved by proper choice of the parameters $A$ and $B$. For example, the choice $A=0.75$ and $B=1$ yields $\tau^{*}=0.211$ and $\tau^{*}=0.212$ respectively in cases (II) and (III).

REMARK 3.3. We observe that the stability region obtained depends on the selection of Lyapunov function(al) and any modification of this function(al) either in terms of functions used or the parameters included in the functional may produce a different portion of the stability region of the system. It is the observation of the authors that, in general, no two Lyapunov function(al)s produce the same stability region for the system under consideration. At this juncture, we draw the attention of the readers to the normalisation of $y$ in (3.2). Theorem 3.1 may be tried without a normalisation 
of $y$ to obtain a different estimation on the delay $\tau$. For example, Cases I and II of Theorem 3.1 with a nonnormalised $y$ assume the following form:

Case I (a) $(D-b \gamma / 2)>0, B d y-A b \gamma y^{*} / 2>0$ and

$$
\tau^{*}=\min \left\{\frac{2 A \beta}{B C}, \frac{1}{2}\left(\frac{A_{1}}{B_{1}}+\frac{A_{2}}{B_{2}}-\sqrt{\left(\frac{A_{1}}{B_{1}}-\frac{A_{2}}{B_{2}}\right)^{2}+\frac{4 C_{1}^{2}}{B_{1} B_{2}}}\right)\right\},
$$

where $A_{1}=A(D-b \gamma / 2), B_{1}=B c D / 2,2 C_{1}=A a L-B c \alpha_{2}, A_{2}=B d-A b \gamma / 2$ and $B_{2}=B c\left[\left(D+a\left(1+y^{*}\right)+b \gamma\right) M+b \gamma+a L^{2}\right] / 2$.

Case II (a) $A(D-b \gamma / 2-a L / 2)-B c \alpha_{2} / 2>0$ and $B d-A b \gamma / 2-A a L / 2-$ $B C \alpha_{2} / 2>0$ with

$$
\begin{aligned}
& \tau^{*}=\min \left\{\frac{A(2 D-b \gamma-a L)-B c \alpha_{2}}{B c D}, \frac{2 A \beta}{B c},\right. \\
& \left.\frac{2 B d-A b \gamma-A a L-B c \alpha_{2}}{B c\left[\left(D+a\left(1+y^{*}\right)+b \gamma\right) M+b \gamma+a L^{2}\right]}\right\} \text {. }
\end{aligned}
$$

For these estimations, we get for the parametric values of Example 3.2, for Case I (a), $\tau_{1}=0.5507, \tau_{2}=0.6724$ and $2 A \beta / B C=6$, and hence $\tau^{*}=0.5507=\tau_{1}$.

Thus in this case we have obtained a better estimation on $\tau$ than Case I of Theorem 3.1. But we notice that Case II (a) above cannot be applied here as the first condition

$$
\left(D-\frac{b \gamma}{2}-\frac{a L}{2}\right)-\frac{B c \alpha_{2}}{2}>0
$$

fails to hold for the above parametric values.

It would be interesting to know what changes the nonnormalised term $y$ brings in the other cases. Further, the functional used in the proof of Theorem 3.1 is not unique and an enthusiastic reader may try different ones for a few more stability conditions.

REMARK 3.4. Theorem 3.1 gives the length of the delay for which the system is stable when the system has a finite carrying capacity $(d>0)$. But for large $d$, we have from the equilibrium solutions (2.4) that $y^{*}$ takes small values and eventually $y^{*} \rightarrow 0$ as $d \rightarrow \infty$. From the conditions on the parameters of the system in Theorem 3.1, we can see that $\tau^{*}$ increases for increasing $d$, but is always bounded (by $c L-\gamma-D$ ), eventually tending to $c U\left(x_{0}\right)-\gamma-D$. But when $d \rightarrow \infty, y^{*} \rightarrow 0$ which means that the consumer population becomes extinct. This is surely not desirable. This means that when the system has a definite carrying capacity it can tolerate a delay in the growth response of the species and under the conditions of Theorem 3.1, the species will survive. 
We may notice that Assumptions $\left(A_{3}\right)-\left(A_{4}\right)$ restrict the choice of the uptake function very much for global stability of the positive equilibrium. In order to consider a more general class of uptake functions we shall now try to establish global stability of the positive equilibrium solution of (2.1) employing the following condition which is a special case of a Lipschitz condition. We recall that the existence and uniqueness of the solutions for (2.1) are ensured by conditions weaker than a Lipschitz condition on $U$ as noted in Section 2.

For this we suppose that there exists a $k>0$ such that

$$
\left|U(x)-U\left(x^{*}\right)\right| \leq k\left|x-x^{*}\right|, \quad \text { for all } x \in \mathbb{R}_{+} .
$$

Letting $x_{1}(t)=x(t)-x^{*}, \quad y_{1}(t)=y(t)-y^{*}$ and $U_{1}\left(x_{1}(t)\right)=U(x(t))-U\left(x^{*}\right)$, we rewrite system (2.1) as

$$
\begin{aligned}
x_{1}^{\prime}(t)= & -D x_{1}(t)-a U(x(t)) y_{1}(t)-a y^{*} U_{1}\left(x_{1}(t)\right) \\
& +b \gamma \int_{0}^{\infty} f(s) y_{1}(t-s) d s, \\
y_{1}^{\prime}(t)= & \left(y_{1}(t)+y^{*}\right)\left[c U_{1}\left(x_{1}(t-\tau)\right)-d y_{1}(t)\right] .
\end{aligned}
$$

We now have the following result.

THEOREM 3.5. Assume that the delay kernel $f$ satisfies $\left(\mathrm{A}_{2}\right)$ and the uptake function $U_{1}$ satisfies $\left(\mathrm{A}_{1}\right)$ and $(3.15)$. The equilibrium solution $(0,0)$ of $(3.16)$ is globally asymptotically stable for

$$
0 \leq \tau<\tau^{*}=\min \left\{\frac{D-c k-a k y^{*}}{\left(D+a y^{*}\right) c k}, \frac{d+a \bar{\alpha}-b \gamma}{(a L+b \gamma) c k}\right\},
$$

provided $D-c k-a k y^{*}>0$ and $d+a \bar{\alpha}-b \gamma>0$, in which $\bar{\alpha}=\min _{x \geq x} \cdot\{U(x)\}$.

Proof. We consider the functional, $V(t)=V_{1}(t)+V_{2}(t)$, where

$$
V_{1}(t)=\left|x_{1}(t)\right|+\left|\log \left(\frac{y_{1}(t)+y^{*}}{y^{*}}\right)\right|+b \gamma \int_{0}^{\infty} f(s) \int_{t-s}^{1}|y(u)| d u d s
$$

and

$$
\begin{aligned}
V_{2}(t)= & c k\left[D \int_{t-\tau}^{t} d s \int_{s}^{t}\left|x_{1}(u)\right| d u+a L \int_{t-\tau}^{t} d s \int_{s}^{t}\left|y_{1}(u)\right| d u\right. \\
& +a y^{*} \int_{t-\tau}^{t} d s \int_{s}^{1}\left|U_{1}\left(x_{1}(u)\right)\right| d u \\
& \left.+b \gamma \int_{t-\tau}^{1} d s \int_{0}^{\infty} f(z) \int_{s-z}^{1}\left|y_{1}(u)\right| d u d z\right] .
\end{aligned}
$$


Consider

$$
\begin{aligned}
D^{+} V_{1}(t) \leq & -D\left|x_{1}(t)\right|-a U(x(t))\left|y_{1}(t)\right|-a y^{*}\left|U_{1}\left(x_{1}(t)\right)\right| \\
& +c\left|U_{1}\left(x_{1}(t-\tau)\right)\right|+b \gamma\left|y_{1}(t)\right|-d\left|y_{1}(t)\right| .
\end{aligned}
$$

Now we have

$$
\begin{aligned}
\left|U_{1}\left(x_{1}(t-\tau)\right)\right| \leq & k\left|x_{1}(t-\tau)\right|=k\left|x_{1}(t)-\int_{t-\tau}^{t} x_{1}^{\prime}(s) d s\right| \\
= & k \mid x_{1}(t)-\int_{t-\tau}^{t}\left[-D x_{1}(s)-a U(x(s)) y_{1}(s)-a y^{*} U_{1}\left(x_{1}(s)\right)\right. \\
& \left.+b \gamma \int_{0}^{\infty} f(z) y_{1}(s-z) d z\right] d s \mid \\
\leq & k\left|x_{1}(t)\right|+k\left[D \int_{t-\tau}^{t}\left|x_{1}(s)\right| d s+a L \int_{t-\tau}^{t}\left|y_{1}(s)\right| d s\right. \\
& \left.+a y^{*} \int_{t-\tau}^{t}\left|U_{1}\left(x_{1}(s)\right)\right| d s+b \gamma \int_{t-\tau}^{t} \int_{0}^{\infty} f(z)\left|y_{1}(s-z)\right| d z d s\right] .
\end{aligned}
$$

Thus we have

$$
\begin{aligned}
D^{+} V_{1}(t) \leq & -\left(D-c k-a k y^{*}\right)\left|x_{1}(t)\right|-(d+a U(x)-b \gamma)\left|y_{1}(t)\right| \\
& +c k\left[D \int_{t-\tau}^{t}\left|x_{1}(s)\right| d s+a L \int_{t-\tau}^{t}\left|y_{1}(s)\right| d s\right. \\
& \left.+a y^{*} \int_{t-\tau}^{t}\left|U_{1}\left(x_{1}(s)\right)\right| d s+b \gamma \int_{t-\tau}^{t} \int_{0}^{\infty} f(z)\left|y_{1}(s-z)\right| d z d s\right] .
\end{aligned}
$$

Now

$$
\begin{aligned}
D^{+} V_{2}(t)= & c k\left[D\left|x_{1}(t)\right| \tau+a L\left|y_{1}(t)\right| \tau+a y^{*}\left|U_{1}\left(x_{1}(t)\right)\right| \tau+b \gamma\left|y_{1}(t)\right| \tau\right] \\
& -c k\left[D \int_{t-\tau}^{t}\left|x_{1}(s)\right| d s+a L \int_{t-\tau}^{t}\left|y_{1}(s)\right| d s+a y^{*} \int_{t-\tau}^{t}\left|U_{1}\left(x_{1}(s)\right)\right| d s\right. \\
& \left.+b \gamma \int_{t-\tau}^{t} \int_{0}^{\infty} f(z)\left|y_{1}(s-z)\right| d z d s\right]
\end{aligned}
$$

Using (3.17) and (3.18) we have after some simplifications,

$$
\begin{aligned}
D^{+} V(t) \leq & -\left(D-c k-a k y^{*}\right)\left|x_{1}(t)\right|-(d+a U(x)-b \gamma)\left|y_{1}(t)\right| \\
& +c k\left(D+a k y^{*}\right) \tau\left|x_{1}(t)\right|+c k(a L+b \gamma) \tau\left|y_{1}(t)\right| \\
= & -\left(D-c k-a k y^{*}-c k\left(D+a k y^{*}\right) \tau\right)\left|x_{1}(t)\right| \\
& -(d+a U(x)-b \gamma-c k(a L+b \gamma) \tau)\left|y_{1}(t)\right| .
\end{aligned}
$$

The negative definiteness of $D^{+} V(t)$ follows from the hypotheses. The remainder of the proof may be completed employing standard arguments. 
Notice in this case that we have not normalised any variables here. The purpose is only to introduce different Lyapunov functionals and techniques.

The delay-free system We now consider a special case of (2.1), namely, the system

$$
\begin{aligned}
& x^{\prime}(t)=D\left(x_{0}-x(t)\right)-a U(x(t)) y(t)+b \gamma \int_{0}^{\infty} f(s) y(s) d s, \\
& y^{\prime}(t)=-(\gamma+D) y+c U(x(t))-d y^{2}(t)
\end{aligned}
$$

which is obtained by letting the delay parameter $\tau=0$ in (2.1). The system (3.19) is studied in [7]. Notice that the equilibria of (2.1) and (3.19) are the same and thus a unique positive equilibrium of (3.19) is given by (2.4) under the conditions (2.3) again. Observing that the parametric conditions of Theorem 3.1 are valid for the case $\tau=0$ as well, we now establish sufficient conditions for the global asymptotic stability of the positive equilibrium solution of (3.19). We shall not provide the details of the proof as it may be obtained by a modification of the Lyapunov functional used in the proof of Theorem 3.1 and appropriate manipulation of the terms. We also notice that the condition $\left(\mathrm{A}_{3}\right)$ on $U$ is no longer required here. Thus, we have the following result.

THEOREM 3.6. Assume that the delay kernel $f$ satisfies $\left(\mathrm{A}_{2}\right)$, and the uptake function $U_{1}$ satisfies $\left(\mathrm{A}_{1}\right)$ and $\left(\mathrm{A}_{4}\right)$. The positive equilibrium solution $\left(x^{*}, y^{*}\right)$ of (3.19) is globally asymptotically stable for $\tau=0$ provided there exist positive constants $A$ and $B$ such that the following conditions hold: $A_{1}=A\left(D-b \gamma y^{*} / 2\right)>0$, $A_{2}=B d y^{*}-A b \gamma y^{*} / 2>0$ and $A_{1} A_{2}>C_{1}{ }^{2}$, where $2 C_{1}=A a y^{*} L-B c \alpha_{2}$.

We now give examples to compare Theorem 3.6 with some known results ([7]).

EXAMPLE 3.7. Consider (3.19) with $D=1.3, a=8, b=0.8, \gamma=2, c=7.6$ and $d=10$. That is,

$$
\begin{aligned}
& x^{\prime}(t)=(1.3)\left(x_{0}-x(t)\right)-8 U(x(t)) y(t)+(1.6) \int_{0}^{\infty} f(s) y(t-s) d s, \\
& y^{\prime}(t)=-(3.3) y(t)+(7.6) U(x(t))-10 y^{2}(t),
\end{aligned}
$$

in which $x_{0} \approx 1.0923$ and $U(x)=x /(1+x)$. Clearly, $\left(x^{*}, y^{*}\right)=(1,1 / 20)$ and $U\left(x^{*}\right)=1 / 2$. It is easy to see that all the hypotheses of Theorem 3.6 are satisfied for the choice of $A=5, B=1$ and hence $\left(x^{*}, y^{*}\right)$ is globally asymptotically stable by virtue of Theorem 3.6. It may be observed that the parametric conditions of both Theorems 4.1 and 4.3 of [7] are violated, and hence they are not applicable here. 
EXAMPLE 3.8. Consider the system

$$
\begin{aligned}
& x^{\prime}(t)=\left(x_{0}-x(t)\right)-20 U(x(t)) y(t)+2 \int_{0}^{\infty} f(s) y(t-s) d s, \\
& y^{\prime}(t)=-5 y(t)+16 U(x(t))-2 y^{2}(t)
\end{aligned}
$$

in which $D=1, a=20, b=0.5, \gamma=4, c=16, d=2, x_{0}=22$ and $U(x)=$ $x /(3+x)$. Clearly, $\left(x^{*}, y^{*}\right)=(9,7 / 2)$ and $U\left(x^{*}\right)=3 / 4$. Since $D-b \gamma y^{*} / 2<0$, Theorem 3.6 cannot be applied here, while both Theorems 4.1 and 4.3 of [7] ensure the global asymptotic stability of $\left(x^{*}, y^{*}\right)$ in this case.

It is clear from the above examples that Theorem 3.6 is independent of Theorems 4.1 and 4.3 of [7].

Thus our study adds one more result on global stability in this area.

\section{Some special cases}

4.1. System with infinite carrying capacity Theorem 3.5 motivates us to study the stability of the following chemostat-like model under the influence of the time delay $\tau>0$ in the growth response:

$$
\begin{aligned}
& \frac{d x(t)}{d t}=D\left(x_{0}-x(t)\right)-a U(x(t)) y(t)+b \gamma \int_{0}^{\infty} f(s) y(t-s) d s, \\
& \frac{d y(t)}{d t}=-(\gamma+D) y(t)+c y(t) U(x(t-\tau)) .
\end{aligned}
$$

System (4.1) is obtained from (2.1) for the choice $d=0$ which implies that the carrying capacity of the environment no longer influences the dynamics of the system. All the terms (4.1) have the same meaning and definition as in (2.1). The conditions $\left(A_{1}\right)$ and $(3.15)$ on the uptake function and $\left(A_{2}\right)$ on the delay kernel $f$ are assumed to hold in this section also.

Under these assumptions, it is shown in $[19,29]$ that the solutions to (4.1) do exist, and are unique and continuable in their maximal intervals of existence with appropriate initial conditions (for example, (2.2)). It is further shown that the solutions of (4.1) are dissipative and bounded.

It may be noticed that $\left(x_{0}, 0\right)$ is an equilibrium solution of $(4.1)$ also. But we are interested in the survival of the species and look for a positive equilibrium solution which, if it exists, should satisfy

$$
D x^{*}+a U\left(x^{*}\right) y^{*}-b \gamma y^{*}=D x_{0} \quad \text { and } \quad c U\left(x^{*}\right)=\gamma+D .
$$


By Assumption $\left(\mathrm{A}_{1}\right), x^{*}>0$ exists if $\gamma+D / c<L$ and $y^{*}$ given by $y^{*}=$ $D\left(x_{0}-x^{*}\right) /\left(a U\left(x^{*}\right)-b \gamma\right)$ exists and is positive if $\left(x_{0}-x^{*}\right)\left(a U\left(x^{*}\right)-b \gamma\right)>0$. Since $c \leq a$ and $b \in(0,1)$, we have $a U\left(x^{*}\right)-b \gamma>0$ whenever $x^{*}>0$ exists. Therefore $y^{*}>0$ exists if $x_{0}>x^{*}$.

In this context we assume that

$$
\frac{\gamma+D}{c}<L \text { and } x_{0}>x^{*}
$$

where $x^{*}$ is such that $U\left(x^{*}\right)=\gamma+D / c$, which implies the existence of a positive equilibrium solution $\left(x^{*}, y^{*}\right)$ for (4.1).

In the following result, we obtain a lower bound for $\tau>0$ for which the positive equilibrium solution $\left(x^{*}, y^{*}\right)$ remains globally asymptotically stable. As the proof of this result is quite similar to that of Theorem 3.5 , we only state the result.

THEOREM 4.1. The equilibrium solution $\left(x^{*}, y^{*}\right)$ of (4.1) is globally asymptotically stable for

$$
0 \leq \tau<\tau^{*}=\min \left\{\frac{D-c k-a k y^{*}}{\left(D+a y^{*}\right) c k}, \frac{a \bar{\alpha}-b \gamma}{(a L+b \gamma) c k}\right\},
$$

provided $D-c k-a k y^{*}>0$ and $a \bar{\alpha}-b \gamma>0$, in which $\bar{\alpha}=\min _{x \geq x^{*}}\{U(x)\}$ and $k$ is the Lipschitz constant defined in (3.15).

REMARK 4.2. System (4.1) is considered by He and Ruan in [19]. It may be noticed that the technique adopted by $\mathrm{He}$ and Ruan in [19] enforces the restrictions

$$
\tilde{T}_{f}=\int_{0}^{\infty} f(s)\left[\exp ^{(\gamma+D) s}-1\right] d s<\infty \quad \text { and } \quad T_{f}^{*}=\int_{0}^{\infty} f(s)\left[1-\exp ^{-\gamma_{1} s}\right] d s<\infty
$$

$\gamma_{1}=c-\gamma-D$ on the delay kernels, in addition to $\left(\mathrm{A}_{2}\right)$. This reduces the choice of delay kernels $f$ in [19]. Moreover, the terms $\tilde{T}_{f}$ and $T_{f}^{*}$ are included in the estimation of the delay $\tau$ which implies the direct influence of the delay kernel on $\tau$ of [19]. This clearly establishes that our estimate is quite different from that obtained in [19].

The following example compares the lengths of the delay parameter estimated by Theorem 4.1 above and [19, Theorem 4.1].

EXAMPLE 4.3. Consider the system

$$
\begin{aligned}
& x^{\prime}(t)=8\left(x_{0}-x(t)\right)-22 U(x(t)) y(t)+\int_{-\infty}^{t} f(t-s) y(s) d s, \\
& y^{\prime}(t)=-10 y(t)+20 U(x(t-\tau)),
\end{aligned}
$$


in which $D=8, \gamma=2, b=0.5, x_{0}=11$ and $U(x)=x /(10+x)$. Then $\left(x^{*}, y^{*}\right)=$ $(10,0.8)$ and $U\left(x^{*}\right)=0.5$ with $k=0.1$. For these parametric values, the length of the delay given by Theorem 4.1 for which the system is globally asymptotically stable is $\tau^{*}=0.2172$ while $\left[19\right.$, Theorem 4.1] estimates the delay to be $\tau^{*}=0.162$.

The following example supports the claim in Remark 4.2.

EXAMPLE 4.4. Consider the system

$$
\begin{aligned}
& x^{\prime}(t)=2(14-x(t))-14 U(x(t)) y(t)+\int_{-\infty}^{t} f(t-s) y(s) d s, \\
& y^{\prime}(t)=-6 y(t)+8 U(x(t-\tau)),
\end{aligned}
$$

in which $\gamma=4, b=0.75$ and $U(x)=x /(4+x)$ with $f(s)=4 e^{-4 s}$. Then $\left(x^{*}, y^{*}\right)=(12,8 / 15)$ and $U\left(x^{*}\right)=0.75$ with $k=1 / 8$. Clearly all the conditions of Theorem 4.1 are satisfied, yielding $\tau^{*}=0.00704$. Therefore Theorem 4.1 ensures the global asymptotic stability of $(12,8 / 15)$ for $0 \leq \tau<\tau^{*}=0.00704$. When

$$
\int_{0}^{\infty} f(s)\left[\exp ^{(\gamma+D) s}-1\right] d s \rightarrow \infty
$$

[19, Theorem 4.1] cannot be applied here.

We understand here that the conditions provided by He and Ruan may yield larger values for $\tau$ in some cases but this may not be achieved without severely restricting the other parameters of the system. At the same time, the parametric conditions of Theorem 4.1 are easy to work with and Theorem 4.1 is applicable in many cases as we have seen in the above examples.

REMARK 4.5. No monotonicity or differentiability conditions are placed on the uptake function $U$ in Theorem 4.1 unlike as in earlier studies ( $[1-7,10,16-20,29,30])$. The Michaelis-Menten uptake function, namely, $U(x)=a x /(m+x)$, is chosen as the one suitable for studies in a chemostat. But one cannot expect all the microorganisms in nature to be of the same form. Some more specific forms of uptake functions studied by researchers which have experimental support are given below (see [31] for more details).

(1) Consider the function $U(x)=m x^{2} /\left(a_{1}+x\right)\left(b_{1}+x\right)$, in which $m_{1}, a_{1}$ and $b_{1}$ are positive constants.

(2) Another well-known example of an uptake function is the following:

$$
U(x)=\frac{m}{1+b / x+x / c}, \quad m, b, c>0,
$$

which is an example of a nonmonotonically increasing uptake function. 
All the earlier studies $([1-7,10,16-20,29,30]$, see also the discussion before Theorem 3.5) have defined their uptake functions to follow the properties of the above functions which are at least obviously bounded and continuously differentiable.

Now consider the function

$$
U(x)=\frac{x^{\alpha}}{\omega+x^{\beta}}, \quad \omega>0,0<\alpha \leq \beta .
$$

Termed a generalised Michaelis-Menten uptake function (see [22]), this function satisfies a Lipschitz condition for $\alpha \geq 1$ and fails to satisfy the condition at $x=0$ when $0<\alpha<1$. Observe also that $x=0$ is the only such point at which the condition fails to be satisfied. The behaviour of all the functions that are mentioned above as experimentally proved uptake functions in a chemostat can be studied from this function. In [22], the authors established the existence and uniqueness of solutions to the system under consideration. The system considered in [22] deals with a distributed delay in the growth equation of $y$ in place of a fixed delay $\tau$ as in (2.1). Further, the stability of equilibria with such uptake functions is also discussed in [22]. However, in the present case we require a Lipschitz-type condition (3.15) on the system (4.1) (or (2.1)) to establish sufficient conditions for stability. We observe that it is the selection of Lyapunov function that imposes this condition on $U$.

Hence we remark that our results apply to a more general class of delay kernels and uptake functions than those envisaged by [19].

4.2. System without material recycling We shall now consider the case when the death of the species is attributed only to the washout. That is, the washout is fast enough that natural death numbers are insignificant $(\gamma=0)$. In such a case, (4.1) further reduces to

$$
\begin{aligned}
& x^{\prime}(t)=D\left(x_{0}-x(t)\right)-a U(x(t)) y(t), \\
& y^{\prime}(t)=-D y(t)+c U(x(t-\tau)) .
\end{aligned}
$$

System (4.3), a chemostat model with time delay in growth response was studied in $[4,16]$. It is known that solutions to (4.3) do exist and are positive and bounded under suitable initial conditions (see (2.2)).

System (4.3) has a positive equilibrium solution under the conditions $D<c L$ and $x_{0}>x^{*}$, where $x^{*}>0$ is such that $U\left(x^{*}\right)=D / c$ (see the discussion above (4.2)).

Recently, Beretta and Kuang [4] estimated the delay length for which the positive equilibrium solution $\left(x^{*}, y^{*}\right)$ is globally asymptotically stable assuming $c=a$ in (4.3). We observe that Theorem 4.1 enables us to study the stability of $\left(x^{*}, y^{*}\right)$ in this special case also. We now present an example to compare the results of both Theorem 4.1 when $\gamma=0$ and Theorem 3.1 of [4]. 
EXAMPLE 4.6. Consider the system

$$
\begin{aligned}
& x^{\prime}(t)=3\left(x_{0}-x(t)\right)-5 U(x(t)) y(t), \\
& y^{\prime}(t)=-3 y(t)+5 U(x(t-\tau)),
\end{aligned}
$$

in which $D=3, a=5=c, x_{0}=5.5$ and $U(x)=x /(3+x)$. Then $\left(x^{*}, y^{*}\right)=$ $(4.5,1)$ with $k=2 / 15$ and $\bar{\alpha}=3 / 5$. Now from Theorem 4.1 , we have after some calculations, $\tau^{*}=5 / 16$. This further implies that $\left(x^{*}, y^{*}\right)$ is globally asymptotically stable for $0 \leq \tau \leq 5 / 16$ by virtue of Theorem 4.1 .

By appropriate scaling we obtain $(9 / 11,2 / 11)$ as the corresponding equilibrium solution of system (1.1) of [4]. The length of the delay for which this equilibrium is stable is estimated to be $\tau^{*}=0.002115$, employing [4, Theorem 3.1]. It is clear in this case that the estimate on the length of the delay parameter given by Theorem 4.1 here is much larger than the one given by [4, Theorem 3.1]. Further, we have noticed that the procedure suggested in [4] for the estimation of $\tau^{*}$ is tedious as it involves a large number of calculations. Moreover, the length of the delay parameter given by [4, Theorem 3.1] depends on the bounds on the solutions of the system which, in turn, depend on the delay parameter itself, which is not the case with Theorem 4.1 above.

REMARK 4.7. The choices $D=1, x_{0}=1$ and $a=c=1$, (or by appropriate scaling) reduce (4.3) to the considerations of Beretta and Kuang ([4]). Noting that the equilibrium solution $\left(x^{*}, y^{*}\right)$ satisfies $1-x^{*}-y^{*}=0$ and $U\left(x^{*}\right)=1$, the length of the delay $\tau^{*}$ in this case is given by $\tau^{*}=\min \left\{1-k x^{*} /\left(1+y^{*}\right) k, 1 / L k\right\}$, using Theorem 4.1. Thus if we can find $k$ defined in (3.15) such that $k x^{*}<1$, then for $0 \leq \tau<\tau^{*}$, the system (1.1) of [4] is globally asymptotically stable. It may be seen that this estimate on $\tau^{*}$ is different from the one obtained in [4]. Further, no differentiability conditions are placed on $U$ unlike as in [4].

\section{Discussion}

It is interesting to note that delay-independent results (with stability, no matter how large the delay is) would severely restrict the parameters of the system. Due to these restrictions, delay-independent stability results become less applicable. Moreover, these results are of little interest given that increasing time delays always destabilises the system [11,13-15]. Hence it is worthwhile discussing results which depend on the delay (that is, delay-dependent stability results) for which the equilibrium is stable. This is the starting point of our discussion in the present paper in which we have proposed a mathematical model involving a discrete delay in the growth response of the consumer species and a distributed delay in the nutrient recycling. Employing a Lyapunov functional technique, we have obtained various sets of sufficient conditions 
on the parameters, estimating the length of the delay for which the positive equilibrium is globally asymptotically stable. We have not placed any a priori conditions on the parameters for the stability of the positive equilibrium of the delay-free system. Thus our method applies to the delay-free system as well (that is, when $\tau=0$ ).

\section{Acknowledgements}

This research is supported by a grant from the Foundation for Scientific Research and Technological Innovation - A division of Sri Vadrevu Seshagiri Rao Memorial Charitable Trust, Hyderabad-500 035, India.

The authors wish to thank the anonymous referees for many helpful suggestions.

\section{References}

[1] E. Beretta and G. I. Bischi, "Stability and Hopf bifurcations in some nutrient-species models with nutrient recycling and time lags", in Biomedical Modelling and Simulation (ed. J. C. Baltzer), (Scientific Publishing Co., IMACS, Basel, 1989) 175-181.

[2] E. Beretta, G. I. Bischi and F. Solimano, "Stability in a chemostat model with delayed nutrient recycling", J. Math. Biol. 28 (1990) 99-111.

[3] E. Beretta and Y. Kuang, "Convergence results in a well known delayed predator-prey system", $J$. Math. Anal. Appl. 204 (1996) 840-853.

[4] E. Beretta and Y. Kuang, "Global stability in a well known delayed chemostat model", Commun. Appl. Anal. 4 (2000) 147-165.

[5] E. Beretta and Y. Takeuchi, "Global stability for chemostat equations with delayed nutrient recycling”, Nonlin. World 1 (1994) 291-306.

[6] E. Beretta and Y. Takeuchi, "Qualitative properties of chemostat equations with time delays: boundedness, local and global stability", Differential Equations Dyn. Syst. 2 (1994) 19-40.

[7] E. Beretta and Y. Takeuchi, "Qualitative properties of chemostat equations with time delays II", Differential Equations Dyn. Syst. 2 (1994) 263-288.

[8] T. A. Burton, Stability and Periodic Solutions of Ordinary and Functional Differential Equations (Academic Press, New York, 1985).

[9] J. Caperon, "Time lag in population growth response of Isochrysis galbana to a variable nitrate environment", Ecology 50 (1969) 188-192.

[10] S. P. Ellermeyer, "Competition in the chemostat: Global asymptotic behaviour of a model with delayed response in growth", SIAM J. Appl. Math. 54 (1994) 456-465.

[11] L. H. Erbe. H. I. Freedman and V. Sree Hari Rao, "Three species food chain models with mutual interference and time delays", Math. BioSciences 80 (1986) 57-80.

[12] P. Fergola, L. Jiang and Z. Ma, "On the dynamics of a chemostat model with delayed nutrient recycling", Int. J. Appl. Math. Comp. Sci. 10 (2000) 81-96.

[13] H. I. Freedman and V. Sree Hari Rao, "Trade-off between mutual interference and time lags in predator-prey systems", Bull. Math. Biol. 45 (1983) 991-1004.

[14] H. I. Freedman and V. Sree Hari Rao, "Stability criteria for a system involving two time delays", SIAM J. Appl. Math. 46 (1986) 552-560. 
[15] H. I. Freedman, V. Sree Hari Rao and K. J. Lakshmi, "Stability, persistence and extinction in a predator-prey system with discrete and continuous time delays", WSSIAA 1 (1992) 221-238.

[16] H. I. Freedman, J. W. H. So and P. Waltman, "Coexistence in a model of competition in the chemostat incorporating discrete delays", SIAM J. Appl. Math. 49 (1989) 859-870.

[17] H. I. Freedman and F. Yang. "Competing predators for a prey in a chemostat model with periodic nutrient input", J. Math. Biol. 29 (1991) 715-732.

[18] H. I. Freedman and Y. Xu, "Model of competition in the chemostat with instantaneous and delayed nutrient recycling", J. Math. Biol. 31 (1993) 513-527.

[19] X.-Z. He and S. Ruan, "Global stability in chemostat-type plankton models with delayed nutrient recycling". J. Math. Biol. 37 (1998) 253-271.

[20] X.-Z. He, S. Ruan and H. Xia, "Global stability in chemostat-type equations with distributed delays", SIAM J. Math. Anal. 29 (1998) 681-696.

[21] R. M. Nisbet and W. S. C. Gurney, Modelling fluctuating populations (John Wiley \& Sons, Chichester, New York, 1982).

[22] V. Sree Hari Rao and P. Raja Sekhara Rao, "Global stability of chemostat models involving time delays", Differential Equations. Dyn. Syst. 8 (2000) 1-28.

[23] V. Sree Hari Rao and P. Raja Sekhara Rao, "Global stability of chemostat models involving time delays and zones of no activation", Canad. Appl. Math. Quart. 9 (2001) 273-300.

[24] V. Sree Hari Rao and P. Raja Sekhara Rao, "Global stability of chemostat models involving time delays and wall growth", Nonlinear Anal. Real World Appl. 5 (2004) 141-158.

[25] V. Sree Hari Rao and P. Raja Sekhara Rao, "Mathematical models of microbial populations and issues concerning stability", Chaos Solitons Fractals 23 (2005) 657-670.

[26] V. Sree Hari Rao and P. Raja Sekhara Rao, "Oscillations induced by time lags in limited nutrientconsumer dynamic models", Chaos Solitons Fractals 25 (2005) 11-23.

[27] V. Sree Hari Rao and P. Raja Sekhara Rao, "Mathematical models and stabilizing bio-control mechanisms for microbial populations in a cultured environment", Chaos Solitons Fractals (in press).

[28] V. Sree Hari Rao, P. Raja Sekhara Rao and K. Venkata Ratnam, "Parameter estimation using dynamic optimization in limited nutrient-consumer hybrid dynamical systems", Special issue on Hybrid Dynamical Systems, Nonlinear Anal. 64 (2006) 263-287.

[29] S. Ruan, "The effect of delays on stability and persistence in plankton models", Nonlinear Anal. 24 (1995) 575-585.

[30] S. Ruan and X.-Z. He, "Global stability in chemostat-type competition models with nutrient recycling", SIAM J. Appl. Math. 58 (1998) 170-192.

[31] H. L. Smith and P. Waltman. The Theory of the chemostat (Cambridge University Press, Cambridge, 1985).

[32] P. Waltman, "Coexistence in chemostat-like models", Rocky Mountain J. Math. 20 (1990) 777-807.

[33] G. S. K. Wolkowicz, H. Xia and S. Ruan, "Competition in the chemostat: a distributed delay model and its global asymptotic behaviour". S/AM J. Appl. Math. 55 (1997) 1281-1310.

[34] S. Yuan, M. Song and M. Han, "Direction and stability of bifurcating periodic solutions of a chemostat model with two distributed delays", Chaos Solitons Fractals 21 (2004) 1109-1123. 